\title{
Relational Properties in Interaction Aesthetics: The Ubiquitous Music Turn
}

\author{
Damián Keller \\ NAP, \\ Federal University of Acre \\ Rio Branco, AC, Brazil \\ dkeller@ccrma.stanford.edu \\ Maria Helena de Lima \\ Federal University of \\ Rio Grande do Sul \\ Porto Alegre, RS, Brazil \\ helena.lima@ufrgs.br
}

\author{
Nuno Otero \\ Linnaeus University \\ Kalmar \\ Sweden \\ nuno.otero@Inu.se
}

\author{
Victor Lazzarini \\ National University of Ireland \\ Maynooth, Co. Kildare \\ Ireland \\ victor.lazzarini@nuim.ie
}

Marcelo Soares Pimenta

Federal University of

Rio Grande do Sul

Porto Alegre, RS, Brazil

mpimenta@inf.ufrgs.br
Marcelo Johann

Federal University of

Rio Grande do Sul

Porto Alegre, RS, Brazil

johann@inf.ufrgs.br

\author{
Leandro Costalonga \\ Federal University of \\ Espírito Santo
}

São Mateus, ES, Brazil leandro.costalonga@ufes.br

\begin{abstract}
Taking as a point of departure recent theoretical advances in Interaction Design and HumanComputer Interaction (Löwgren 2009), we discuss a body of knowledge gathered in Ubiquitous Music practices (Keller et al. 2011a) during the last six years. New concepts and methods have been proposed to describe aspects of the ideation and materialisation of experiences with technology. Pliability (Löwgren 2007) and anchoring (Keller et al. 2010) are two of the multiple design qualities that surfaced in interaction design that impact information technology creative practices. We present results of experiments addressing creativity support for ubiquitous music making through the time tagging metaphor (Radanovitsck et al. 2011). The studies serve to exemplify how relational properties can be integrated within creativity-centred design. Our research indicates that sonic relational properties may provide affordances for proto-musical phenomena. We discuss the theoretical and methodological implications of this proposal highlighting its impact on everyday musical creativity.
\end{abstract}

Ubiquitous music. Interaction aesthetic proto-musical qualities.

\section{INTRODUCTION}

In 2005, Udsen \& Jørgensen stated: 'at present, the aesthetic turn is not a full-fledged shift in paradigm. However, it is undoubtedly an indication of a new awareness of the wide-ranging dimensions of interaction between humans and computers.' We have reasons to believe this situation has changed, particularly within the practices of interaction design.

Interaction aesthetics is surfacing as a strong alternative to mainstream human-computer interaction theories and methods (Hällnas Redström 2002, Löwgren 2007, Löwgren 2009, Redström 2007, Stolterman 2008, Udsen \& Jørgensen 2005). Löwgren (2009) and Stolterman (2008) propose a shift in focus from task-oriented, utilitarian approaches to human-centred and experience-centred methods, described as a 'rational, disciplined, designerly way.' (Stolterman 2008). Redström (2007) suggests that a central idea is the need to create a richer relation to computational things, through the exploration of:
- engagement rather than efficiency,

- temporal patterns of behaviour;

- alternative forms of design that challenge expectations;

- user identities, cultural contexts and traditions, within specific design domains;

- innovative material combinations.

Despite the significant theoretical advances in interaction aesthetics, how to approach the variety of methodological issues raised by this perspective on technology is still an open question. In one of the initial studies in this area, Redström (2001) endorsed a radical change of focus: "how to design for living with, rather than just using, computational technology.' To design for everyday life involves more than supporting people to accomplish certain tasks effectively. Designs for usability and functionality are not sufficient. This broader view of interaction explores aspects for which the traditional usability assessment methods are 
incapable of providing useful information. New techniques are necessary.

From an aesthetically aware perspective, Stolterman (2008) proposes the following methods: '(i) precise and simple tools or techniques, (ii) frameworks that do not prescribe but that support reflection and decision-making, (iii) individual concepts that are intriguing and open for interpretation and reflection on how they can be used, (iv) high-level theoretical and/or philosophical ideas and approaches that expand design thinking but do not prescribe design action.' Sketching and prototyping (Buxton 2007) are examples of item (i).

These techniques reduce the development cycle ensuing multiple iterations through the design process. Instead of prescribing solutions for welldefined problems, interaction aesthetics techniques may involve the application of design patterns (Borchers \& Mühlhäuser 1998), design actions (De Bruijn \& Spence 2008), and interaction metaphors to handle open-ended research problems. Personas, scenarios, probes and affordances are tools that provide inspiration to deal with situated issues, fostering reflection and understanding of the implications of each design decision. Thus, situated experience, consensual rationale and reflective practice are surfacing as key aspects of the interaction aesthetics approach to design.

\section{GOALS OF UBIMUS DESIGN}

Ubiquitous Music research (Keller et al. 2011a) has also targeted aspects of interaction design that have not been approached from the mainstream human-computer interaction $(\mathrm{HCl})$ perspectives. Ubiquitous music deals with systems of human agents and material resources that afford musical activities through sustainable creativity support tools. Viewing Redström's (2007) interaction aesthetics proposal from a ubiquitous music perspective, we see a convergence of interests and methods: engagement, temporal patterns of behaviour, alternative forms of design with innovative material combinations, and user identities inserted in cultural contexts. Despite these common themes, there are two ubiquitous music design concepts that have not been considered in interaction aesthetics: creative potentials and everyday creativity. Let us review the convergent approaches first.

Engagement has surfaced as one of the constructs that impact creativity outcomes (Keller et al. $2011 b)$. This factor is particularly relevant when the activities involve intense social interaction (Pimenta et al. 2012). Engagement may encompass multiple mechanisms related to personal (Keller \& Capasso
2000) and social aspects of the creative activity (Lima et al. 2012).

Temporal patterns of behaviour have gained increased relevance through the study of creative activity. A new focus on activities carried in everyday settings has opened the door to the study of everyday musical creativity (Pinheiro da Silva et al. 2013b) with promising applications in the field of education (Lima et al. 2012).

Regarding alternative forms of design and innovative material combinations, these lines of research were already present in ecocompositional practices (Keller 2000) but have gained new strength with the introduction of the technique of repurposing within ubiquitous music systems design (Flores et al. 2010). Multimodality (Keller 2004) also seems to be an emerging common theme that may be further developed by the exploration of proto-musical design qualities.

User identities, cultural contexts and traditions have been partially explored within the domain of ubiquitous music design. Brazilian creativity traits were introduced in interaction design by Pimenta et al. (2012). This line of research may be expanded by cross-cultural and ethnographic studies, although the current emphasis seems to be on the impact of user identities on aspects of everyday creativity.

A focus that is missing from current research efforts in interaction aesthetics is the socially distributed nature of creative activity. This aspect is featured in the application of communities of practice within ubiquitous music research (Pimenta et al. 2012).

Ubiquitous music research may also provide a contribution to interaction aesthetics by expanding the design goals to account for creative phenomena. While existing approaches synthesised in Redström (2007) - focused on alternative forms of design and innovative material combinations, ubiquitous music techniques target the increase of creative potentials (DiLiello \& Houghton 2008). Thus, one objective is the empowerment of participants in creative actions, rather than just the implementation of creative products or artefacts. Hummels \& Overbeeke (2010: 2) stated that 'design is about being-in-theworld.' Paraphrasing, we can say that ubiquitous music design is about being creative in the everyday world.

Ubiquitous music design decisions are materialised as technological prototypes that afford but do not enforce creative behaviour. This proposal is rooted in the Brazilian dialogical education movement (Freire 1970, Lima et al. 2012). Relational properties result both from agent-object interactions 
and agent-agent (or social) interactions. When the social dynamic is non-hierarchical, creative products and processes may not necessarily fit within the division of labour applied in industry: users may become co-creators. In this sense, the dialogical approach has strongly influenced the participatory design movement (Ehn 1988). Given that the technological prototype is not the end product, ubiquitous music research focuses on creative activities in everyday contexts. Therefore, the site-specific creative experience - rather than digital musical instruments, instrumental virtuosity or isolated sound objects (Schaeffer 1977) - is the material for study. Because creative ubiquitous musical experiences occur in everyday contexts and involve ordinary people, experiments are done outside of the institutionalised spaces for music making. Hence, another objective of the ubiquitous music design process is the support of manifestations of everyday creativity (Richards et al. 1988), defined as the processes and products that are both innovative and socially relevant but that do not attain status of artworks.

\section{RELATIONAL PROPERTIES IN UBIMUS DESIGN: METHODOLOGICAL IMPLICATIONS}

Given the radical shift set forth by the interaction aesthetics research program, existing approaches to interaction design are being reformulated bringing new issues to the foreground. Relational properties - such as pliability (Löwgren), anchoring (Keller et al. 2010) capture the tight agent-object interaction dynamic that has been at the centre of the embedded-embodied approaches to cognition (Gibson 1979). In line with the enactive perspective (Di Paolo et al. 2007), these design qualities emerge as a result of mutual adaptations between agents and objects. Because they demand the active engagement of an agent, relational properties cannot be 'attached' to objects. They can only be experienced 'in the act'. There are several methodological implications of adopting relational properties as theoretical constructs.

Firstly, design experiments must take into account both subjective and material constraints and opportunities. Experiments that adopt closed epistemic spaces - where subjects are given a task to complete in laboratory settings and the results measure their efficiency in completing the task - do not provide information on the ecosystem's support for the emergence of relational properties. In this case, the material resources are chosen by the experimenter and may not fulfill the creative needs of the subject. This is an example of the problem identified by ubiquitous music research, 'autoreferentiality of the theoretical-experimental construct' (Keller et al. 2011b): the experimental situation does not take into account the conditions of real-world settings. Relational properties may be absent from a laboratory task but may be accessible when participants are interacting on site.

Second, the adoption of a tool at the initial stages of the design process may forego the emergence of relational properties. The potential of the agentobject ecosystem to support relational properties is not enough. This potential has to be materialised in products and behaviours. Given that the behaviours depend on personal factors (including both cognitive and social resources), understanding the creative profiles of the subjects is an integral part of the design process. From a ubiquitous music perspective, this procedural limitation is defined as 'early domain restriction' (Keller et al. 2011b).

Third, assessments purely based on products do not provide a complete picture. There is a rich literature of product evaluation methods in creativity studies (Mumford et al. 2011). Creative products give reliable information on creative outcomes. This information can be enhanced by the analysis of domain-specific products. Nevertheless, despite its untapped potential, this retrospective approach has an epistemological limitation: products and procedures are usually not equivalent. Asynchronous creative activities furnish procedural data not easily recoverable from the finished products. So this data needs to be collected during the creative activity (Coughlan \& Johnson 2006). Contrastingly, synchronous creative proposals (such as improvisatory musical practices) fuse products and processes into a single experience. In this case, the assessment of the product imposes the same requirements as the assessment of the creative process: time-based methods. Hence, whether considering synchronous or asynchronous creative activities, if a relational property emerges as a by-product, it may not be discernible through the analysis of the finished product but it may be observable while the product is being made or used.

Furthermore, while only creative products are not enough to enhance the knowledge of an aesthetically aware design, just creative processes may be insufficient to assess the level of support for creative behaviour. Creative potentials are externalised as creative actions, which may yield creative by-products. The creative magnitude of these by-products can only be assessed in relation to a specific social context (or social niche - Keller 2012). This context is shaped by the life histories of the participants of the creative experiences. Therefore, evaluations of technological support for creative behaviour involve: (i) assessments of byproducts, (ii) observations of situated behaviours, and (iii) analyses of the profiles of the participants. 
As Mumford et al. (2011) suggest, creativity evaluation methods are necessarily multidimensional. Ubiquitous music research is no exception.

This section discussed the procedural implications of the adoption of relational properties in ubiquitous music experimental research. To overcome the limitations of previous methodological approaches, three strategies were identified: (1) use of realworld resources and conditions, (2) furnishing potential material resources that match the profiles of the participants, (3) adopting time-based multidimensional evaluation methods, including byproducts' profiles, situated behaviours and subjective evaluations. Given the complementary goals of interaction aesthetics and ubiquitous music research and the methodological implications of adopting relational properties as experimental variables in ubiquitous music research, what are the consequences of the application of the Ubimus agenda in interaction aesthetics? Would Ubimus concepts provide a sharper focus to aesthetically aware design? Let us take one example of a ubiquitous music design outcome to discuss the implications for interaction design: time tagging.

\section{TIME TAGGING}

The results presented in this section summarise a long cycle of design processes that involved several iterations of proposals, implementations, experiments and critiques (Keller et al. 2010). A first prototype - Drum! (Kuhn 2011) - targeted tapping as a synchronous musical activity on portable devices. A musical design pattern was explored in this first mobile prototype (Flores et al. 2010).

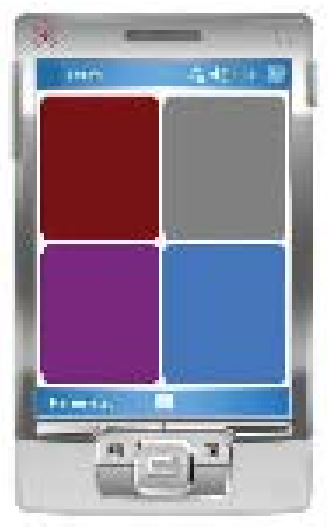

Figure 1: Percussive prototype Drum! (Kuhn 2011)

A series of brainstorming sessions involving rhythmic playing were carried out with a PDA device. From these sessions, a direct relation between the device's input capabilities and the musical potentialities emerged: by tapping the touchscreen with the stylus, percussive sounds could be elicited. This mode of interaction is very close to the patterns of behaviour observed in primates (Arcadi et al. 1998). So these initial results indicated that an interaction metaphor involving sonic events elicited by percussive behaviour could be worth pursuing.

A second series of prototypes - mixDroid (Radanovitsck et al. 2011) - extended percussive actions to found sounds. In mixDroid, the interaction metaphor is based on synchronous sequential access to each of the sampled sounds stored in memory. Sound data is sent to a mixing buffer that constitutes the tagged timeline. While the user accesses the samples, the contents of the buffer are being played. This mechanism provides sonic cues that serve to shape the temporal anchoring process. The user listens to the sounds on the timeline and the samples being played provide cues for the new sounds that will enter the mix. Finally, the new sound entering the timeline is tagged when the participant presses the interface's soft buttons.

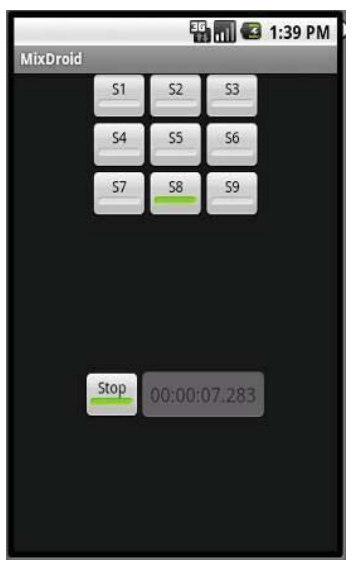

Figure 2: Time tagging in mixDroid 1.0 (Keller et al. 2010, Radanovitsck et al. 2011)

The mixDroid prototypes materialise the concept of time tagging as an interaction metaphor for creative musical activity and incorporate creative activities as both exploratory and decision-making strategies. Time tagging is a multimodal metaphor that applies anchoring to sound mixing. Anchoring serves as a cognitive mechanism for linking constraints of the external structure of the environment to constraints on cognitive operations (Keller et al. 2010). Metaphors for interaction based on anchoring can take advantage of direct couplings between physical and conceptual operations. More generally, time tagging defines a process by which a set of unordered virtual elements or processes is layered onto an abstract one-dimensional structure - a tagged timeline.

Time tagging was proposed as a solution to the computational burden of the visually oriented approaches to audio mixing (Keller et al. 2010). 
This interaction metaphor provided a fertile ground for experiments on creative musical experiences in everyday environments by both musicians and nonmusicians (Pinheiro da Silva et al. 2012). In experiments with naive users doing simple mixing activities on portable devices, the time tagging metaphor showed better performance when compared with the traditional tape-editing metaphor (featured in most desktop audio editing software) (Radanovitsck et al. 2011).

As an initial validation process of the time-tagging metaphor, we used the mixDroid prototype for the creation of a complete musical work (Keller et al. 2009). A musician with full musical training and over 20 years of experience with music technology was chosen for the study. This choice was based on the need to integrate actual expert usage within the development cycle. The objective was to test whether the interaction technique would be suitable for a complete sound mixing cycle: from the initial state - defined as a collection of unordered sound samples, to the final state - a time-based organised set of sounds. Although sound quality and accurate synchronisation were not key aspects in this initial trial, we ensured support for standard PCM formats and sample-based synchronisation. The procedure consisted of several mixing sessions. MixDroid was used in the emulation mode on a laptop computer and was activated through pointing and clicking with an optical mouse. Several dozens of sound samples were used, with durations ranging from a less than a second to approximately two minutes. The sonic palette was extended by means of ecological modeling (Keller \& Truax 1998) and asynchronous granular synthesis (Keller \& Rolfe 1998, Truax 2002). Resonant filtering was used to modify the spectral qualities of the sounds, keeping the macrotemporal cues of the sources untouched. The temporal structure of the creative product was moulded by the temporal distribution of the sonic materials (frog calls). The result was a sevenminute stereo sound work: Green Canopy On The Road (Keller et al. 2009).

After completing a first validation phase involving expert usage, we focused on the demands of naive participants in everyday settings. Two complementary studies were conducted. A second study (Pinheiro da Silva et al. 2013a) comprised creative activities in public settings (at a shopping centre, at a busy street and at an area featuring animal sounds) and in private settings (at the home of each participant and at a studio facility). Six subjects participated in 47 mixing sessions using samples collected at two outdoors sites - one featuring urban sounds and the other highlighting animal sounds - and in studio settings. Creativity support was evaluated by means of a creativeexperience protocol encompassing six factors: productivity, expressivity, explorability, enjoyment, concentration, and collaboration (Carroll et al. 2009). Outdoor sessions yielded higher scores in productivity, explorability, concentration and collaboration when compared to studio sessions. Compound effects of sound sample type and activity location were observed in the explorability factor when animal sounds were used. Similar effects were detected on explorability, productivity and concentration when urban sounds were employed.

A third study (Keller et al. 2013a) made use of recorded vocal samples created by the participants. In order to untangle the effects of place and activity type, three conditions were studied: place (including domestic and commercial settings); activity type (such as imitative mixes and original creations); and body posture (realizing the mix while standing or sitting). Ten subjects took part in an experiment encompassing 40 interaction sessions using mixDroid. Subjects created mixes and assessed their experiences through a modified version of the protocol applied in the previous study (CSI-NAP, Keller et al. 2011b). Increased scores in the explorability and collaboration factors were observed in the activities taking place in domestic settings.

The results show the impact of the venue on the assessment for creativity support. The outdoor spaces were preferred by the participants of the second study. Domestic settings got slightly higher ratings in the third study. The profile of the subjects impacted the results of the third study but this trend was not confirmed by the second study's results. The data analysis highlighted a limitation in the assessment technique proposed by (Carroll et al. 2009): the concentration factor needs to be partitioned into two independent measures related to cognitive load and engagement (Bryan-Kinns 2013, Lima et al. 2012).

\section{IMPLICATIONS FOR AESTHETICALLY AWARE DESIGN APPROACHES}

The results reported in the previous section highlight the complementary goals of interaction aesthetics and ubiquitous music research and the design implications of adopting relational properties as experimental variables in ubiquitous music research.

First, adopting complete cycles of creative activity within the design process gives access both to creative products and procedural data. If the introduced technology supports results that are relevant and innovative, then a baseline condition of the design has been achieved. The published musical work Green Canopy: On The Road served 
as this first-stage validation. To avoid isolating the design activity from the local resources, real-world contexts are necessary.

Second, the two studies conducted in everyday settings, comprising musicians and non-musicians, provided a profile of the limitations and advantages of the time tagging metaphor regarding agentobject and agent-agent interactions. The studies pointed to a close relationship between environmental variables, personal variables and creative activity. Both the environmental settings and the profile of the participants impacted the evaluation of the interaction metaphor. While the effect of the place factor was conclusive, the studies showed the need to refine the assessment tool. Engagement and cognitive load need to be detangled.

\section{CONCLUSIONS AND PROPOSALS FOR FUTURE STUDIES}

This paper establishes a common ground for ubiquitous music research and interaction aesthetics. Methods used in interaction aesthetics have been fruitfully explored in the context of ubiquitous music problems, providing examples of the use of domain-neutral strategies within domainspecific research. Knowledge transfer in the other direction may be less intuitive, but we have provided examples of interaction designs that found a firmer ground for experiments by considering domain-specific proposals and results. More specifically, the research we have been pursuing during the last six years in the Ubiquitous Music Group highlights the usefulness of focusing on relational properties in interaction design.

Taking as example the use of relational properties in ubiquitous music design, we identified three strategies to deal with aesthetically aware proposals: (1) employing real-world resources and conditions, (2) furnishing potential material resources that match the profiles of the participants, (3) adopting time-based multidimensional evaluation methods, including byproducts' profiles, situated behaviours and subjective evaluations. Having established a common ground for ubiquitous music concepts and interaction aesthetics constructs, we presented three examples of time tagging to support tapping in activities involving sample mixing and percussive sound synthesis. We discussed the pros and cons of the design choices from the experimental data reported in (Radanovitsck et al. 2011, Keller et al. 2011b, Keller et al. 2013, Pinheiro da Silva et al. 2012, Pinheiro da Silva et al. 2013a).

The time tagging metaphor provides support for aesthetically aware approaches to musical interaction design. Restating the question regarding interaction aesthetics, would the time tagging concept provide a sharper focus to aesthetically aware design? To gauge the relevance of this proposal, this issue needs to be placed within a broader perspective. We propose that time tagging belongs to a family of phenomena that can be characterised as being proto-musical. Dissanayake (2008: 175) describes proto-musical behaviours as the altered and unusual facial expressions, sounds, and head and body movements that mothers use in early interaction with their infants.[...] In early interactions, mothers modify their affinitive signals to infants as if to draw the greatest attention to them - that is, to make them maximally effective as unambiguous communication and maximally affecting emotionally.' She argues that 'the cognitive capacities and emotional sensitivities that are used in human music [...] emerged for good evolutionary reasons, from affinitive mechanisms in interactions that evolved gradually between ancestral mothers and infants as early as two million years ago, long before music.' The antecedents of these capacities and sensitivities can be found in the ritualised behaviours of other animals. These proto-musical behaviours employ and are built upon neural substrates and hormonal mechanisms for social affiliation and coordination that already existed in non-human primates. They may have been essential for the survival of helpless human infants (altriciality) and for the reproductive success of mothers. Dissanayake (2008) suggests that through cultural development, these biological capacities and sensitivities became the temporal arts, music being their most illustrative manifestation. By adopting proto-musicality as an experimental construct, we may be able to provide evolutionary and ecological grounding for sonic related design qualities.

Without discarding the aesthetic issues identified by Redström (2007), Arvola (2010) or Hummels \& Overbeeke (2010), we suggested two aesthetically aware design goals that are consistent with ubiquitous music findings: (1) the empowerment of participants through enhanced creative potentials, (2) the support for manifestations of everyday creativity. These goals are closely related to the application of proto-musicality in interaction design and find support in Lowgrëns approach to the aesthetics of design: 'If computing is understood in the everyman's sense of the word (information technology embedded in everyday life), then the aesthetics of computing refers to the sensual/perceptual qualities of experiencing information technology' (Löwgren 2002: 24, in Bertelsen \& Fishwick 2002). We suggest that protomusicality may be a useful construct for future experimental studies of the aesthetic qualities of creative technological experiences. 


\section{REFERENCES}

Arcadi, A., Robert, D., and Boesch, C. (1998) Buttress drumming by wild chimpanzees: Temporal patterning, phrase integration into loud calls, and preliminary evidence for individual distinctiveness. Primates $39 \quad$ (4), 505-518. (Doi: 10.1007/BF02557572.)

Arvola, M. (2010) Interaction design qualities: Theory and practice. In Proceedings of the 6th Nordic Conference on Human-Computer Interaction: Extending Boundaries (NordiCHI 2010) (pp. 595-598). ACM, New York, NY. (ISBN: 978-160558-934-3.)

Bertelsen, O. W. and Fishwick, P (eds.), (2002) Aesthetic Computing [Dagstuhl Seminar Report N ${ }^{\circ}$ 348]. Schloss Dagstuhl, Wadern, Germany.

Borchers, J., and Mühlhäuser, M. (1998) Design patterns for interactive musical systems. IEEE MultiMedia 5 (3), 36-46. (Doi: 10.1109/93.713303.)

Bryan-Kinns, N. (2013) Mutual engagement and collocation with shared representations. International Journal of Human-Computer Studies 71 (1), 76-90. (Doi: 10.1016/j.ijhcs.2012.02.004.)

Buxton, W. (2007) Sketching User Experiences: Getting the Design Right and the Right Design. Elsevier / Morgan Kaufmann, New York, NY. (ISBN: 9780123740373.)

Carroll, E. A., Latulipe, C., Fung, R., and Terry, M. (2009) Creativity factor evaluation: Towards a standardized survey metric for creativity support. In Proceedings of the Seventh ACM Conference on Creativity and Cognition (pp. 127-136). ACM, Berkeley, CA. (ISBN: 978-1-60558-865-0.)

Coughlan, T., and Johnson, P. (2006) Interaction in creative tasks. In Proceedings of the SIGCHI Conference on Human Factors in Computing Systems (pp. 531-540). ACM, New York, NY. (ISBN: 1-59593-372-7.)

De Bruijn, O., and Spence, R. (2008) A new framework for theory-based interaction design applied to serendipitous information retrieval. ACM Transactions on Computer-Human Interaction 15 (1), 5:1-5:38. (Doi: 10.1145/1352782.1352787.)

DiLiello, T. C., and Houghton, J. D. (2008) Creative potential and practised creativity: Identifying untapped creativity in organizations. Creativity and Innovation Management 17 (1), 37-46. (Doi: 10.1111/j.1467-8691.2007.00464.x.)

Di Paolo, E. A., Rohde, M., and Jaegher, H. D. (2010) Horizons for the enactive mind: Values, social interaction, and play. In J. R. Stewart, $\mathrm{O}$. Gapenne and E. A. Di Paolo (eds.), Enaction: Toward a New Paradigm for Cognitive Science (pp. 33-88). Cambridge, MA: MIT Press.
Dissanayake, E. (2008) If music is the food of love, what about survival and reproductive success?. Musicae Scientiae 12 (1 supplement), 169-195. (Doi: 10.1177/1029864908012001081.)

Ehn, P. (1988) Work-Oriented Design of Computer Artifacts. Arbetslivscentrum, Stockholm, Sweden. (ISBN: 91-86158-45-7.)

Freire, P. (1970) Pedagogy of the Oppressed. Continuum, New York. NY.

Gibson, J. J. (1979) The Ecological Approach to Visual Perception. Houghton Mifflin, Boston, MA. (ISBN: 0898599598.)

Hallnäs, L., and Redström, J. (2002) From use to presence: On the expressions and aesthetics of everyday computational things. ACM Transactions on Computer-Human Interaction (ToCHI) 9 (2), 106-124. (Doi: 10.1145/513665.513668.)

Hummels, C., and Overbeeke, K. (2010) Special issue editorial: Aesthetics of interaction. International Journal of Design, 4 (2), 1-2.

Keller, D. (2012) Sonic Ecologies. In A. R. Brown (ed.), Sound Musicianship: Understanding the Crafts of Music (pp. 213-227). Newcastle upon Tyne, UK: Cambridge Scholars Publishing. (ISBN: 978-1-4438-3912-9.)

Keller, D. (2004) Paititi: A Multimodal Journey to El Dorado. Doctoral Thesis in Musical Arts, Stanford University.

Keller, D. (2000) Compositional processes from an ecological perspective. Leonardo Music Journal, 55-60. (Doi: 10.1162/096112100570459.)

Keller, D., Capasso, A., Tinajero, P., Flores, L. V., and Pimenta, M. S. (2009) Green Canopy: On The Road [Ubiquitous Music Work]. In Proceedings of XII Brazilian Simposium on Computer Music (SBCM 2009). SBC, Recife, PE.

Keller, D., Ferreira da Silva, E., Pinheiro da Silva, F., Lima, M. H., Pimenta, M. S., and Lazzarini, V. (2013a) Everyday musical creativity: An exploratory study with vocal percussion. In Proceedings of the National Association of Music Research and PostGraduation Congress - ANPPOM, Natal, RN.

Keller, D., Flores, L. V., Pimenta, M. S., Capasso, A., and Tinajero, P. (2011) Convergent trends toward ubiquitous music. Journal of New Music Research $40 \quad$ (3), 265-276. (Doi: 10.1080/09298215.2011.594514.)

Keller, D., Lima, M. H., Pimenta, M. S., and Queiroz, M. (2011a) Assessing musical creativity: material, procedural and contextual dimensions. In Proceedings of the National Association of Music Research and Post-Graduation Congress ANPPOM (pp. 708-714), Uberlândia, MG. 
Keller, D., Pinheiro da Silva, F., Ferreira da Silva, E., Lazzarini, V., and Pimenta, M. S. (2013b) Opportunistic design of ubiquitous music systems: the impact of anchoring on creativity. In $E$. Ferneda, G. Cabral and D. Keller (ed.), Proceedings of the XIV Brazilian Symposium on Computer Music (SBCM 2013), Brasília, DF.

Keller, D., Pinheiro da Silva, F., Giorni, B., Pimenta, M. S., and Queiroz, M. (2011b) Spatial tagging: an exploratory study. In Proceedings of the 13th Brazilian Symposium on Computer Music, Vitória, ES.

Keller, D., and Truax, B. (1998) Ecologically based granular synthesis. In Proceedings of the International Computer Music Conference (pp. 117120). MPublishing, University of Michigan Library, Ann Arbor, MI.

Keller, D., and Rolfe, C. (1998) The corner effect. In Proceedings of the XII Colloquium on Musical Informatics. Gorizia: AIMI.

Kuhn, C. (2011). Drum! [Software prototype]. LCM, Porto Alegre, RS.

Lima, M. H., Keller, D., Pimenta, M. S., Lazzarini, V., and Miletto, E. M. (2012) Creativity-centred design for ubiquitous musical activities: Two case studies. Journal of Music, Technology and Education 5 (2), 195-222. (Doi: 10.1386/jmte.5.2.195_1.)

Löwgren, J. (2007) Pliability as an experiential quality: exploring the aesthetics of interaction design. Artifact 1 (2), 85-95. (Doi: 10.1080/17493460600976165.)

Löwgren, J. (2009) Toward an articulation of interaction esthetics. New Review of Hypermedia and Multimedia 15 (2), 129-146. (Doi: 10.1080/13614560903117822.)

Mumford, M. D., Hester, K., and Robledo, I. (2011) Methods in creativity research: Multiple approaches, multiple methods. In M. D. Mumford (ed.), Handbook of Organizational Creativity (pp. 39-64). Elsevier Science, Waltham, MA. (ISBN: 9780080879109.)
Pimenta, M. S., Miletto, E. M., Keller, D., and Flores, L. V. (2012) Technological support for online communities focusing on music creation: Adopting collaboration, flexibility and multiculturality from Brazilian creativity styles. In N. A. Azab (ed.), Cases on Web 2.0 in Developing Countries: Studies on Implementation, Application and Use. IGI Global Press, Vancouver, BC:. (ISBN: 1466625155.)

Pinheiro da Silva, F., Keller, D., Ferreira da Silva, E., Lazzarini, V., and Pimenta, M. S. (2013a) Creativity in everyday settings: The impact of anchoring. In Proceedings of the IV Ubiquitous Music Workshop (IV UbiMus), Porto Alegre, RS.

Pinheiro da Silva, F., Keller, D., Ferreira da Silva, E., Pimenta, M. S., and Lazzarini, V. (2013b) Everyday musical creativity: Exploratory study of ubiquitous musical activities. Música Hodie 13 (1), 64-79.

Pinheiro da Silva, F., Pimenta, M. S., Lazzarini, V., and Keller, D. (2012) Time tagging in its niche: Engagement, explorability and creative attention. In Proceedings of the III Ubiquitous Music Workshop (III UbiMus), São Paulo, SP.

Radanovitsck, E. A. A., Keller, D., Flores, L. V., Pimenta, M. S., and Queiroz, M. (2011) mixDroid: Time tagging for creative activities. In Proceedings of the XIII Brazilian Symposium on Computer Music (SBCM 2011), Vitória, ES.

Redström, J. (2007) Aesthetic concerns. In G. Giaglis and P. Kourouthanassis (ed.), Pervasive Information Systems (pp. 197-209), Armonk, NY.

Schaeffer, P. (1977) Traité des objets musicaux: Essai interdisciplines. Éditions du Seuil, Paris. (ISBN: 9782020026086.)

Stolterman, E. (2008) The nature of design practice and implications for interaction design research. International Journal of Design [Online] 2 (1), 5565. 